\title{
Sensibilidade dos nematóides gastrintestinais de caprinos a anti-helmínticos na mesorregião do Sertão Paraibano ${ }^{1}$
}

\author{
Adriana B. Rodrigues ${ }^{2 *}$, Ana Célia R. Athayde ${ }^{3}$, Onaldo G. Rodrigues ${ }^{3}$, Wilson W. \\ Silva $^{3}$ e Eduardo B. Faria ${ }^{4}$
}

\begin{abstract}
Rodrigues A.B., Athayde A.C.R., Rodrigues O.G., Silva W.W. \& Faria E.B. 2007. [Evaluation of the efficacy of antihelmintics to control gastrointestinal nematodes in goats raised in the state of Paraíba.] Sensibilidade dos nematóides gastrintestinais de caprinos a anti-helmínticos na mesorregião do sertão paraibano. Pesquisa Veterinária Brasileira 27(4):162166. Curso de Pós-Graduação de Medicina Veterinária em Pequenos Ruminantes, Centro de Saúde e Tecnologia Rural, Universidade Federal de Campina Grande, Campus de Patos, 58700970 Patos, PB, Brazil. E-mail: adrianabonfimr@yahoo.com.br

The objective was to evaluate conventional and alternative anthelminthic action on the sensibility of gastrointestinal nematodes in goats. Hundred and twenty goats of both sexes were distributed into 10 groups of 24 animals each (12 males and 12 females) and submitted to treatments of specific anthelminthic composition. The commercial drugs used were moxidectin $0.2 \%$, albendazole, levamisol chlorhydrate, ivermectin and an aqueous extract of the purgative potato (Operculina hamiltonii). The reduction test was applied through egg counts per gram of feces and larval culture to evaluate resistance. The fecal samples were collected in the day when medication was accomplished (day base), at 7,14 and 21 days after treatment. The following results for the reduction of Trichostrongyloidea eggs were obtained: Treatment of the females with Moxidectina reduced the egg counts to $92.8,88.7$ and $89.8 \%$, of the males to $92.6,96.2$ and $98.1 \%$; with Levamisol of the females to $96,97.1$ and $91 \%$, of the males to 85.7, 94,2 and 100\%; with Albendazol of the females 65, 60.3 and 75.4\%, of the males 88.8, 88.8 and 55.5\%; with Ivermectin of the females to $92.2,68.6$ and 70.6\%, of the males to $41.7,73.6$ and 59.7\%; and with the purgative potato of the females to $31.8,34.1$ and $49.4 \%$, of the males to $61.5,80.7$ and $50 \%$. In larval culture Haemonchus, followed by Bunostomum, Trichostrongylus, and Oesophagostomum, were identified even after treatments.
\end{abstract}

INDEX TERMS: Trichostrongyloidea, gastrointestinal helminths, anti-helminthics, egg count.

RESUMO.- As helmintoses gastrintestinais ocupam um lugar de destaque na estatística de problemas sanitários da caprinocultura moderna, criando continuamente novas dificuldades para o seu controle, sendo a resistência a drogas anti-helmínticas fator estratégico limitante para o seu controle. $O$ presen-

\footnotetext{
${ }^{1}$ Recebido em 29 de março de 2006.

Aceito para publicação em 11 de dezembro de 2006.

${ }^{2}$ Pós-Graduando em Medicina Veterinária em Pequenos Ruminantes, Universidade Federal de Campina Grande (UFCG), Centro de Saúde e Tecnologia Rural (CSTR), Rodovia Patos-Teixeira Km 0, Jatobá, Cx. Postal 64, Patos, PB 58700-970, Brasil. "Autor para correspondência: adrianabonfimr@yahoo.com.br

${ }^{3}$ Unidade Acadêmica de Medicina Veterinária, Centro de Saúde e Tecnologia Rural, UFCG (www.cstr.ufcg.edu.br). E-mails: wouflan@hotmail.com, onaldo@cstr.ufcg.edu.br, athayde@cstr.ufcg.edu.br

${ }^{4}$ Curso de Medicina Veterinária, CSTR, UFCG. E-mail: edupqd@ig.com.br
}

te estudo teve o objetivo de verificar a sensibilidade de nematóides gastrintestinais de caprinos a ação de compostos anti-helmínticos, convencionais e alternativos. Foram utilizados 120 animais, de ambos os sexos, sendo distribuídos em grupos de 24 animais cada (12 animais machos e 12 animais fêmeas), totalizando 10 grupos, submetidos, cada grupo ao tratamento com um composto anti-helmíntico específico. As drogas utilizadas nos ensaios foram a moxidectina $0,2 \%$, albendazole, cloridrato de levamisol, ivermectina (drogas convencionais) e extrato aquoso de batata de purga (Operculina hamiltonii). Para avaliar a resistência, aplicou-se o teste de redução na contagem de ovos por grama de fezes (RCOF) e a larvacultura. As amostras fecais foram coletadas no dia em que foi realizada a medicação (dia base), aos 7, 14 e 21 dias após tratamento. Foram obtidos os seguintes resultados para a redução de ovos para a família Trichostrongyloi- 
dea, no tratamento de fêmeas com a moxidectina reduziu 92,8\%, 88,7\% e 89,8\%; nos machos: $92,6 \%, 96,2 \%$ e $98,1 \%$; com o levamisol as fêmeas reduziram 96\%, 97,1\% e 91\%; nos machos: $85,7 \%, 94,2 \%$ e $100 \%$; com o albendazol as fêmeas reduziram $65 \%, 60,3 \%$ e $75,4 \%$; nos machos $88,8 \%, 88,8 \%$ e $55,5 \%$; com a ivermectina reduziram $92,2 \%, 68,6 \%$ e $70,6 \%$; nos machos $41,7 \%$, $73,6 \%$ e $59,7 \%$; com a batata de purga as fêmeas reduziram $31,8 \%, 34,1 \%$ e $49,4 \%$, nos machos $61,5 \%, 80,7 \%$ e $50 \%$. Na cultura de larvas o gênero Haemonchus, seguido de Bunostomum., Trichostrongylus e Oesophagostomum, foram identificados mesmo após os tratamentos.

TERMOS DE INDEXAÇ̃̃O: Trichostrongyloidea, helmintoses gastrintestinais, anti-helmínticos, OPG.

\section{INTRODUÇÃO}

Os caprinos representam uma fonte de proteína de origem animal, o que torna a caprinocultura uma das atividades importantes, do ponto de vista socioeconômico (Vieira 1999). Entretanto, a atividade é limitada, pelo parasitismo desenvolvido por helmintos gastrintestinais (Gopal et al. 1999).

Os prejuízos causados por nematóides gastrintestinais são mais evidentes na região Nordeste. Os principais gêneros encontrados são Haemonchus, Trichostrongylus, Strongyloides, Moniezia, Cooperia, Oesophagostomum, Skrjabinema, Trichuris e Cysticercus. Surtos epizoóticos de Haemoncose e estrongiloidose no Semi-árido paraibano vem aumentando os índices de morbidade e mortalidade do efetivo caprino (Athayde et al. 1996). No nordeste brasileiro, Haemonchus contortus é a espécie de maior prevalência para caprinos e ovinos (Santa Rosa et al. 1986).

A difusão ineficiente no repasse de tecnologias e utilização terapêutica iatrogênica das drogas antiparasitárias em ruminantes tem contribuído para a resistência anti-helmíntica (RA) em várias regiões produtoras do país (Echevarria et al. 1996, Vieira \& Cavalcante 1999).

Vários princípios ativos de anti-helmínticos vêm sendo utilizados no tratamento de nematóides de caprinos, dentre esses, os benzimidazóis, as avermectinas, os imidazotiazóis e as salicilanilicidas (Borges 2003).

A RA é um fenômeno pelo qual uma droga não consegue manter a mesma eficácia terapêutica contra os parasitas, se utilizada nas mesmas condições, após um determinado período de tempo, ou seja, quando uma determinada droga que apresentava redução da carga parasitária acima de 95\% decresce a nível inferior a este valor contra o mesmo organismo decorrido pelo período determinado (Conder \& Campbell 1995).

Os primeiros relatos a cerca da resistência dos helmintos foi frente à aplicação dos benzimidazóis e levamisoles. Em 1981, surgiram as avermectinas, entretanto a resistência ao ivermectin, importante droga deste grupo, tem sido registrada em caprinos parasitados, principalmente por Ostertagia circumcincta e Haemonchus contortus em vários países (Varady et al. 1993, Terril et al. 2001, Gatongi et al. 2003) inclusive no Brasil (Mattos et al. 1997). No Nordeste brasileiro, desde a década de 80 a resistência anti-helmíntica em nematóides de caprinos vem sendo estudada, principalmente no Ceará (Vieira et al. 1989), em Pernambuco e Bahia (Barreto \& Silva 1999).

0 teste de resistência controlado é um dos procedimentos mais confiáveis para a determinação da eficácia antihelmíntica em ruminantes. Testes anti-helmínticos são realizados com infecções induzidas artificialmente, ou em animais portadores de infecções naturalmente adquiridas (GMC 1996).

As cabras têm um metabolismo anti-helmíntico diferente (Conder \& Campbell 1995) e, nesta espécie ocorre a mais baixa biodisponibilidade depois da administração oral. Por conseguinte é suspeitado que RA pareça emergir rapidamente em nematóides infectando cabras, que conseqüientemente podem ser transferidos para as ovelhas (Escudero et al. 1999). Isto já tem acontecido para nematóides resistentes para levamisole e benzimidazoles (Conder \& Campbell 1995) e possivelmente para lactonas macrocíclicas (Escudero et al. 1999).

O objetivo do presente trabalho foi o de avaliar a sensibilidade de nematóides gastrintestinais de caprinos a compostos anti-helmínticos na mesorregião do Sertão paraibano.

\section{MATERIAL E MÉTODOS}

Local de realização do experimento e o período de execução. $\mathrm{O}$ experimento foi desenvolvido em propriedades localizadas no Sertão Paraibano e as análises nos Laboratórios de Doenças Parasitárias dos Animais Domésticos (LDPAD) e Ciências Químicas Biológicas (LCQB) do Centro de Saúde e Tecnologia Rural (CSTR) da Universidade Federal de Campina Grande (UFCG), Campus de Patos. $O$ período de execução da pesquisa foi de agosto de 2004 a janeiro de 2005, perfazendo um total de 180 dias.

Seleção das propriedades. Foram realizadas visitas a algumas propriedades rurais, cadastradas previamente, com o objetivo de coletar fezes e para a obtenção de informações sobre os métodos antiparasitários usados, excepcionalmente, a freqüiência de utilização de drogas anti-helmínticas. Os critérios adotados para a seleção dos animais incluídos neste experimento foram: 1) o diagnóstico positivo para parasitos gastrintestinais pela contagem do número de ovos por grama de fezes (OPG); e 2) a escolha de animais que não teriam sido tratados com qualquer droga anti-helmíntica por um período mínimo e antecedente de 3 meses (90 dias).

Animais utilizados no experimento. Foram utilizados 120 animais (60 machos e 60 fêmeas) com idade variando entre três e oito meses de vida. Os animais foram marcados individualmente, por meio de brincos ou colares e aleatoriamente distribuídos em cinco grupos de doze animais cada: Grupo I tratado com a moxidectina $0,2 \%$; Grupo II tratado com albendazole a $1,9 \%$; Grupo III tratado com o cloridrato de levamisol à 5\%; Grupo IV tratado com a ivermectina $0,08 \%$ e o Grupo $\mathrm{V}$ tratado com extrato aquoso da batata-de-purga (Operculina halmiltonii).

Descrição da posologia empregada nos tratamentos. As doses utilizadas foram aquelas recomendadas pelos fabricantes e pelo LCQB/CSTR/UFCG. A via de administração usada foi a oral em dose única. Ao Grupo I foi administrado moxidectina $0,2 \%$ na dose de $1,0 \mathrm{~mL} / 10 \mathrm{~kg}$; ao Grupo II foi administrado albendazole na dose de $2,0 \mathrm{~mL} / 10 \mathrm{~kg}$; ao Grupo III foi administrado cloridrato de levamisol à $5 \%$ na dose de $1,0 \mathrm{~mL} / 10 \mathrm{~kg}$; ao Grupo IV foi administrada a ivermectina na dose $2,5 \mathrm{~mL} / 10 \mathrm{~kg}$ e ao Grupo $\mathrm{V}$ o extrato aquoso da batata de purga, na dose de $2,5 \mathrm{~mL} / 10 \mathrm{~kg}$.

Amostras de fezes. Amostras fecais foram coletadas diretamente da ampola retal, identificadas em sacos plásticos e mantidas sob 
refrigeração até o processamento da determinação do número de ovos por grama de fezes $(\mathrm{OPG})$ e para a obtenção das larvas. As coletas foram feitas no dia do tratamento (dia base), sete, 14 e 21 dias após tratamento.

Exames parasitológicos. Foram realizadas à contagem do OPG pela técnica descrita por Gordon \& Whitlock (1939) e a realização da larvacultura pela técnica de Roberts e O' Sullivan (1950). Para a identificação das larvas utilizou-se a chave de Keith (1953).

Teste de redução da contagem de ovos por grama de fezes. As médias aritméticas do número de ovos nas fezes, para cada grupo tratado (OPGt), foram calculadas e comparadas com as médias contadas no grupo controle (OPGc). A redução na contagem de ovos nas fezes (RCOF) foi determinada usando a fórmula descrita por Coles et al. (1992):

$$
\begin{aligned}
& \text { RCOF = }[1-(\mathrm{OPGt} / \mathrm{OPGc})] \text { x } 100 \\
& \text { Em que: } \mathrm{RCOF}=\text { teste de redução da contagem de ovos por grama de } \\
& \text { fezes; } \\
& \text { OPGt }=\text { média do número de ovos por grama de fezes do grupo } \\
& \text { de animais tratados; } \\
& \text { OPGc }=\text { média do número de ovos por grama de fezes do grupo } \\
& \text { controle. }
\end{aligned}
$$

A avaliação da efetividade dos fármacos utilizados baseou-se na determinação proposta pelo Grupo Mercado Comum para substâncias químicas, dotados de atividade antiparasitária: segundo os graus é altamente efetivo quando reduz mais que $98 \%$; efetivo 90 98\%; moderadamente efetivo $80-89 \%$; e insuficientemente ativo menos que $80 \%$, não registrável (GMC 1996).

\section{RESULTADOS E DISCUSSÃO}

Constatou-se redução na contagem do OPG nos animais tratados em relação ao grupo controle. No grupo de caprinos fêmeas (Quadro 1), entre os dias 0 e 21 pós-tratamento, o percentual de eficácia variou de $31,8 \%$ a $91,0 \%$; no grupo de caprinos machos (Quadro 2) este percentual variou de $41,7 \%$ a $100 \%$. Ressaltando-se ainda que, nenhum efeito colateral foi observado nos animais dos grupos tratados.

O tratamento de fêmeas com a moxidectina por via oral revelou um percentual de eficácia de $92,8 \%, 88,7 \%$ e $89,8 \%$ aos 07, 14 e 21 dias após o início do tratamento. No entanto, para os grupos de machos de $92,6 \%, 96,2 \%$ e $98,1 \%$. Dados que não corroboram com os de Papadopoulos et al. (2004) que em condições experimentais semelhantes registraram uma eficácia da moxidectina oral de $100 \%$ em caprinos aos 14 dias pós-tratamento e $93,5 \%$ com 56 dias. Os resultados obtidos se

Quadro 1. Média da redução do número de ovos por grama de fezes (OPG) de nematóides gastrintestinais e percentual médio da eficácia de drogas anti-helmínticas em caprinos fêmeas na mesorregião do Sertão Paraibano em diferentes períodos de observação

\begin{tabular}{lcccccccc}
\hline \multirow{2}{*}{$\begin{array}{c}\text { Anti-hel- } \\
\text { míntico }\end{array}$} & $\begin{array}{c}\text { OPG } \\
\text { (dia 0) }\end{array}$ & $\begin{array}{c}\text { OPG } \\
\text { (dia 7) }\end{array}$ & $\begin{array}{c}\text { Eficiência } \\
(\%)\end{array}$ & $\begin{array}{c}\text { OPG } \\
\text { (dia 14) }\end{array}$ & $\begin{array}{c}\text { Eficiência } \\
(\%)\end{array}$ & $\begin{array}{c}\text { OPG } \\
\text { (dia 21) }\end{array}$ & $\begin{array}{c}\text { Eficiência } \\
\text { (\%) }\end{array}$ \\
\hline Moxidectina & 1400 & 100 & 92,8 & 158,3 & 88,7 & 141,7 & 89,8 \\
Levamisole & 1475 & 58,3 & 96,0 & 41,7 & 97,1 & 133,3 & 91,0 \\
Albendazol & 1050 & 366,7 & 65,0 & 416,7 & 60,32 & 258,3 & 75,4 \\
Ivermectina & 425 & 33,3 & 92,2 & 133,3 & 68,6 & 125 & 70,6 \\
Batata de purga & 708,3 & 483,3 & 31,8 & 466,7 & 34,1 & 358,3 & 49,4
\end{tabular}

Quadro 2. Média da redução do número de ovos por grama de fezes (OPG) de nematóides gastrintestinais e percentual médio da eficácia de drogas anti-helmínticas em caprinos machos na mesorregião do Sertão Paraibano em diferentes períodos de observação

\begin{tabular}{lccccccc}
\hline \multirow{2}{*}{$\begin{array}{c}\text { Anti-hel- } \\
\text { míntico }\end{array}$} & $\begin{array}{c}\text { OPG } \\
\text { (dia 0) }\end{array}$ & $\begin{array}{c}\text { OPG } \\
(\text { dia 7) }\end{array}$ & $\begin{array}{c}\text { Eficiência } \\
(\%)\end{array}$ & $\begin{array}{c}\text { OPG } \\
\text { (dia 14) }\end{array}$ & $\begin{array}{c}\text { Eficiência } \\
(\%)\end{array}$ & $\begin{array}{c}\text { OPG } \\
\text { (dia 21) }\end{array}$ & $\begin{array}{c}\text { Eficiência } \\
(\%)\end{array}$ \\
\hline Moxidectina & 450 & 33,3 & 92,6 & 16,7 & 96,2 & 8,3 & 98,1 \\
Levamisole & 291,7 & 41,7 & 85,7 & 16,7 & 94,2 & 0 & 100 \\
Albendazol & 225 & 25 & 88,8 & 25 & 88,8 & 100 & 55,5 \\
lvermectina & 600 & 350 & 41,7 & 158,3 & 73,6 & 241,7 & 59,7 \\
Batata de purga & 216,7 & 83,3 & 61,5 & 41,7 & 80,7 & 108,3 & 50
\end{tabular}

enquadram, segundo preconiza o GMC (1996), que a droga quando é administrada em fêmeas é altamente efetivo e nos machos é insuficientemente ativo, ou seja, não registrável.

No grupo de fêmeas tratadas com o albendazole observou-se um percentual de eficácia de $65,0 \%, 60,3 \%$ e $75,4 \%$, aos 7, 14 e 21 dias e dos machos variou de $88,8 \%, 88,8 \%$ e $55,5 \%$, caracterizando uma droga insuficientemente ativa, ou ainda, não registrável (GMC 1996). Resultados que corroboram com Barreto \& Silva (1999) e Melo et al. (2003) que constataram $79,31 \%$ e 87,5\% de eficácia RCOF 10 dias após tratamento nos estados da Bahia e no Ceará e que não se assemelha com o de Vieira \& Calvacante (1999) que observaram 35,3\% de resistência em rebanhos caprinos no Ceará com o uso de um benzimidazóis (oxfendazol).

O tratamento com o cloridrato de levamisol por via oral em fêmeas apresentou um percentual de eficácia de 96,0\%, $97,1 \%$ e $91,0 \%$ aos 7, 14 e 21 dias após o início do tratamento e nos machos de $85,7 \%, 94,2 \%$ e $100 \%$, caracterizando uma droga moderadamente efetiva nas fêmeas e nos machos altamente efetiva (GMC 1996). Dados que não corroboram com os de Melo et al. (2003) e Vieira \& Cavalcante (1999) que obtiveram prevalência de nematóides resistentes em caprinos aos catorze dias de $75 \%$ e $20,6 \%$ em rebanhos de caprinos do estado do Ceará.

$\mathrm{O}$ uso da ivermectina oral em fêmeas apresentou um percentual de eficácia de $92,2 \%, 68,6 \%$ e $70,6 \%$ aos 7,14 e 21 dias após o início do tratamento e nos machos a redução encontrada foi de $41,7 \%, 73,6 \%$ e 59,7\%, caracterizando uma droga insuficientemente ativa (GMC 1996). Dados similares ao de Gatongi et al. (2003) na África com 59,7\% na redução de ovos em caprinos. No Brasil, Mattos et al. (2004) e Melo et al. $(1998,2003)$ verificaram a eficácia da ivermectina $42,10 \%$ aos 14 dias pós-tratamento em Porto Alegre e 69\% aos 10 dias no Ceará. Dados que discordam com o de Mattos et al. (2003) que observaram eficácia de 93,24\%.

No tratamento realizado nos grupo de fêmeas com o extrato aquoso da batata de purga o percentual de eficácia foi de $31,8 \%, 34,1 \%$ e 49,4\% nos 7, 14 e 21 dias após tratamento, enquanto que nos machos foi de $83,3 \%, 80,7 \%$ e $50 \%$. Dados registrados que se assemelham com de por Almeida (2005) quando trabalhou com extrato alcoólico em caprinos machos e obteve percentual de $85,9 \%$ aos 30 dias pós-tratamento; e com o uso do farelo de batata-de-purga apresentou redução 
Quadro 3. Número de larvas de helmintos observadas por intervalo de 7 dias, em fezes de caprinos na mesorregião do Sertão Paraibano após tratamento anti-helmíntico

\begin{tabular}{|c|c|c|c|c|c|c|c|c|c|c|c|c|c|c|c|c|c|c|c|c|}
\hline \multirow[t]{2}{*}{ Gêneros } & \multicolumn{4}{|c|}{ Moxidectina } & \multicolumn{4}{|c|}{ Albendazol } & \multicolumn{4}{|c|}{ Levamisol } & \multicolumn{4}{|c|}{ Ivermectina } & \multicolumn{4}{|c|}{ Batata de purga } \\
\hline & 0 & 7 & 14 & 21 & 0 & 7 & 14 & 21 & 0 & 7 & 14 & 21 & 0 & 7 & 14 & 21 & 0 & 7 & 14 & 21 \\
\hline Haemonchus & 100 & 100 & 100 & 100 & 100 & 100 & 100 & 100 & 100 & 91 & 88 & 79 & 89 & 98 & 99 & 74 & 92 & 98 & 97 & 99 \\
\hline Bunostomum & 0 & 0 & 0 & 0 & 0 & 0 & 0 & 0 & 0 & 8 & 12 & 0 & 0 & 2 & 1 & 26 & 5 & 2 & 3 & 1 \\
\hline Trichostrongylus & 0 & 0 & 0 & 0 & 0 & 0 & 0 & 0 & 0 & 0 & 0 & 18 & 0 & 0 & 0 & 0 & 1 & 0 & 0 & 0 \\
\hline Cooperia & 0 & 0 & 0 & 0 & 0 & 0 & 0 & 0 & 0 & 0 & 0 & 0 & 0 & 0 & 0 & 0 & 0 & 0 & 0 & 0 \\
\hline Oesophagostomum & 0 & 0 & 0 & 0 & 0 & 0 & 0 & 0 & 0 & 1 & 0 & 3 & 11 & 0 & 0 & 0 & 2 & 0 & 0 & 0 \\
\hline
\end{tabular}

média de $63 \%$ após 30 dias pós-tratamento. E que concordam com os resultados encontrados por Girão et al. (1998) quando trabalhou com infecções naturais de caprinos no Piauí, utilizando pó seco da batata de purga em três concentrações (2, 4 e $6 \mathrm{~g} / \mathrm{kg}$ de peso corpóreo) e constataram que nas concentrações de 2 e $4 \mathrm{~g} / \mathrm{kg}$ de peso corpóreo, não houve redução do $\mathrm{OPG}$ no $7^{\circ}$ dia pós-tratado, e com $6 \mathrm{~g} / \mathrm{kg}$ foi constatado uma redução de 47,0\%. Os resultados obtidos enquadram, segundo preconiza o GMC (1996), a droga é insuficientemente ativa, ou seja, não registrável.

Durante o período de estudo foi observado que $100 \%$ das infecções helmínticas de caprinos eram por helmintos da superfamília Trichostrongyloidea (Quadro 3). O gênero mais prevalente nas coproculturas após 21 dias do início do tratamento foi Haemonchus. Estudos que corroboram com os resultados encontrados no estado do Ceará (Melo et al. 1998, Vieira \& Cavalcante 1999), no nordeste brasileiro (Barreto \& Silva 1999). Provavelmente, esses nematóides desenvolvem resistência mais rápida, devido ao seu alto potencial biótico (Echevarria \& Trindade 1989).

Os resultados obtidos nas larvaculturas estão dispostos no Quadro 3. Aos 21 dias após a vermifugação com a moxidectina $0,2 \%$ foi detectado apenas do gênero Haemonchus. No entanto, Papadopoulos et al. (2004) que em condições experimentais semelhantes revelaram os gêneros de nematóides Trichostrongylus, Teladorsagia, Haemonchus contortus, Chabertia ovina, Oesophagostomum e Bunostomum.

Nas larvaculturas de caprinos tratados com o albendazole observou-se a presença do gênero Haemonchus., Dados quase similares aos relatos por Barreto \& Silva (1999) que obtiveram de Haemonchus, Oesophagostomum, Bunostomun e Tricnhostrongylus, em rebanhos caprinos do Estado da Bahia.

Em caprinos tratados com o cloridrato de levamisol apresentaram larvas do gênero Haemonchus, Trichostrongylus. e Oesophagostomum. Resultados obtidos por Melo et al. (2003) que se assemelham ao estudo.

Após 21 dias observaram-se, nas fezes de animais tratados com a ivermectina, larvas de Haemonchus, e Bunostomun dados que concordam com os relatados por Mattos et al. (2004). Melo et al. (2003) observaram que a ivermectina foi eficaz contra o gênero Oesophagostomum. aos 14 dias, diferentemente dos resultados aqui apresentados.

O gênero Haemonchus foi o único encontrado em animais tratados com o extrato aquoso da batata de purga. 0 estudo da ação de plantas sobre helmintos gastrintestinais é muito escasso o que dificulta a discussão.
Os resultados obtidos no presente estudo indicaram que os nematóides gastrintestinais de caprinos da mesorregião do Sertão Paraibano não são efetivamente sensíveis à ação dos anti-helmínticos moxidectina, albendazol, ivermectina e extrato aquoso de batata de purga, e que são moderadamente sensíveis ao cloridrato de levamisol.

Agradecimentos.- Ao Conselho Nacional de Desenvolvimento Científico e Tecnológico pelo apoio financeiro, e à professora Dra. Maria das Graças Veloso Marinho, Coordenadora do Herbário Caatinga/UFCG, pela preciosa colaboração.

\section{REFERÊNCIAS}

Almeida W.V.F. 2005. Uso de plantas medicinais no controle de helmintos gastrintestinais de caprinos naturalmente infectados. Tese, Universidade Federal de Campina Grande, Paraíba. 63p.

Athayde A.C.R., Nunes R., Araújo M.M. \& Silva W.W. 1996. Surto epizoótico de haemoncose e strongiloidose caprina no semi-árido paraibano. Anais XV Congr. Panam. Ciênc. Vet., Campo Grande, MS, vol.1, p.264.

Barreto M.A. \& Silva J. S. 1999. Avaliação da resistência anti-helmíntica de nematódeos gastrintestinais em rebanhos caprinos do estado da Bahia. Anais XI Seminário Bras. Parasitol. Vet., II Seminário Parasitol. Vet. Países do Mercosul, I Simpósio de Controle Integrado de Parasitos de Bovinos, Salvador, BA, vol.1. 160p.

Borges C.C.L. 2003. Atividade in vitro de anti-helmínticos sobre larvas infectantes de nematódeos gastrintestinais de caprinos, utilizando-se a técnica de coprocultura quantitativa (Ueno, 1995). Parasitol. Latinoam. 58:142-147.

Coles G.C., Bauer C., Borgsteede F.H.M., Geerts S., Klei T.R., Taylor M.A. \& Waller P.J. 1992. World Association for the Advancement of Veterinary Parasitology (WAAVP) methods for the detection of anthelmintic resistance in nematodes of veterinary importance. Vet. Parasitol. 44:35-44.

Conder G.A. \& Campbell W.C. 1995. Chemotherapy of nematode infections of veterinary importance with special reference to drug resistence. Adv. Parasitol. 35:1-83.

Echevarria F.A.M., Borba M.F.S., Pinheiro A.C., Waller P.J. \& Hansen, J.W. 1996. The prevalence of anthelmintic resistanse in nematode parasites of sheep in Southern Latin-America, Brazil. Vet. Parasitol. 62:199-206.

Echevarria F.A.M. \& Trindade G.N.P. 1989. Anthelmintic resistance by Haemonchus contortus to ivermectin in Brazil. Vet. Rec. 124:147-148.

Escudero E., Carceles C.M., Diaz M.S., Sutra J.F., Galtier P. \& Alvinerie M. 1999. Pharmacokinetics of moxidectin and doramectin in goats. Res. Vet. Sci. 67:177-181.

Gatongi P.M., Njoroge J.M., Scott M.E., Ranjan S., Gathuma J.M., Munyua W.K., Cheruiyot H. \& Prichard R.K. 2003. Susceptibility to IVM in a field strain of Haemonchus contortus subjected to four treatments in a closed sheep-goat flock in Kenya. Vet. Parasitol. 110:235-240.

Girão E.S., Carvalho J.H., Lopes A.S., Medeiros L.P. \& Girão R.N. 1998. Avaliação de plantas medicinais com efeito anti-helmíntico para caprinos. Pesquisa em Andamento 78, Embrapa Meio-Norte, Teresina. 9p. 
GMC - Grupo Mercado Comum 1996. Regulamento Técnico para Registros de Produtos Antiparasitários de Uso Veterinário. Decisão no.4/91, resolução no.11/93. Merscosul, Resolução no.76, 1996.

Gopal R.M., Pomroy W.E. \& West D.M. 1999. Resistance of field isolates of Trichostrongylus colubriformis and Ostertagia circumcincta to ivermectin. Int. J. Parasitol. 29:781-786.

Gordon H.M. \& Whitlock H.V. 1939. A new technique for counting nematode eggs insheep faeces. J. Counc. Sci. Ind. Res. 12:50-52.

Keith R.K. 1953. The differential of the infective larval of some common nematode parasites of cattle. Aust. J. Zool. 2:223-230.

Mattos M.J.T., Germer M. \& Castro E.S. 1997. Eficácia do ivermectin sobre endoparasitos de caprinos, no RS. Anais $13^{\circ}$ Congr. Estadual Med. Vet., Gramado, RS, p.198.

Mattos M.J.T., Oliveira C.M.B., Gouvêa A.S. \& Andrade C.B. 2003. Sensibilidade dos nematódeos gastrintestinais de caprinos ao ivermectin na região da Grande Porto Alegre, RS. Acta Scient. Vet. 31(3):155-160.

Mattos M.J.T., Oliveira C.M.B., Gouvêa A.S. \& Andrade C.B. 2004. Haemonchus resistente à lactona macrocíclica em caprinos naturalmente parasitados. Ciência Rural, Santa Maria, 34 (3):879-883.

Melo A.C.F.L., Bevilaqua C.M.L., Villaroel A.S. \& Girão M.D. 1998. Resistência a anti-helmínticos em nematóides gastrintestinais de ovinos e caprinos, no município de Pentecoste, Estado do Ceará. Ciência Animal, Fortaleza, 8:7-11.

Melo A.C.F.L., Reis I.F., Bevilaqua C.M.L., Vieira L.S., Echevarria F.A.M. \& Melo L.M. 2003. Nematódeos resistentes a anti-helmínticos em rebanhos de ovinos e caprinos do estado do Ceará, Brasil. Ciência Rural, Santa Maria, 33:339-344.

Papadopoulos E., Mayrogianni V.S., Ptochos S., Tsakalidis S. \& Fthenakis G.C. 2004. Evaluation of the efficacy of moxidectin in a herd of goats after long-standing consistent use. J. Ruminant Res. 57:271-275.

Roberts F.H.S. \& O'Sullivan J.P. 1950. Methods of egg counts and larval cultures for strongyles infesting the gastrointestinal tract of cattle. Aust. Agric. Res. 1:99-102.

Santa Rosa J., Berne M.E., Johnson E. \& Olander H.J. 1986. Doenças de caprinos diagnosticadas em Sobral, Ceará. Anais da Reunião Técnica do Programa de Apoio à Pesquisa Colaborativa de Pequenos Ruminantes, Sobral, vol.1. Embrapa-CNPC, Sobral, CE, p.235-241.

Terrill T.H., Kaplan R.M., Larsen M., Samples O.M., Miller J.E. \& Gelaye S. 2001. Anthelmintic resistance on goat farms in Georgia: efficacy of anthelmintics agains gastrointestinal nematodes in two selected goat herds. Vet. Parasitol. 97(4):261-268.

Varady M., Praslicka J., Corba J. \& Vesely L. 1993. Multiple anthelmintic resistance of nematodes in imported goats. Vet. Rec. 132:387-388.

Vieira, L.S., Berne M.E.A., Cavalcante A.C.R. \& Menezes R.C.A.A. 1989. Redução do número de ovos por grama de fezes (OPG) em caprinos medicados com anti-helmínticos. Boletim de Pesquisa 11, Embrapa-CNPC, Sobral, CE. 18p.

Vieira L.S. 1999. Epidemiologia e controle da nematodeose gastrintestinal dos caprinos. Anais Congr. Pernambucano Med. Vet., Recife, PE, vol.4, p.123-128.

Vieira L.S. \& Cavalcante A.C.R. 1999. Resistência anti-helmíntica em rebanhos caprinos no Estado do Ceará. Pesq. Vet. Bras. 19:99-103. 